\title{
ALT/AST as an Independent Risk Factor of Gestational Diabetes Mellitus Compared with TG/HDL-C
}

\begin{abstract}
Shuoning Song, (D)' Yuemei Zhang, ${ }^{2}$ Xiaolin Qiao, ${ }^{3}$ Yanbei Duo, ${ }^{1}$ Jiyu $\mathrm{Xu},{ }^{4}$ Zhenyao Peng, ${ }^{5}$ Jing Zhang, ${ }^{6}$ Yan Chen, ${ }^{3}$ Xiaorui Nie, ${ }^{3}$ Qiujin Sun, ${ }^{7}$ Xianchun Yang, ${ }^{7}$ Ailing Wang, ${ }^{8}$ Zechun Lu, ${ }^{8}$ Wei Sun, ${ }^{4}$ Yong Fu, 'Yingyue Dong,' Tao Yuan,' Weigang Zhao'
\end{abstract}

'Department of Endocrinology, Key Laboratory of Endocrinology of Ministry of Health, Peking Union Medical College Hospital, Chinese Academy of Medical Science and Peking Union Medical College, Beijing, People's Republic of China; ${ }^{2}$ Department of Obstetrics, Haidian District Maternal and Child Health Care Hospital, Beijing, People's Republic of China; ${ }^{3}$ Department of Obstetrics, Beijing Chaoyang District Maternal and Child Health Care Hospital, Beijing, People's Republic of China; ${ }^{4}$ Core Facility of Instrument, Institute of Basic Medical Sciences, Chinese Academy of Medical Sciences, School of Basic Medicine, Peking Union Medical College, Beijing, People's Republic of China; ${ }^{5}$ Department of Dean's Office, Haidian District Maternal and Child Health Care Hospital, Beijing, People's Republic of China; ${ }^{6}$ Department of Laboratory, Haidian District Maternal and Child Health Care Hospital, Beijing, People's Republic of China; ${ }^{7}$ Department of Clinical Laboratory, Beijing Chaoyang District Maternal and Child Health Care Hospital, Beijing, People's Republic of China; ${ }^{8}$ National Center for Women and Children's Health, China CDC, Beijing, People's Republic of China

Correspondence: Tao Yuan; Weigang Zhao Department of Endocrinology, Key Laboratory of Endocrinology of Ministry of Health, Peking Union Medical College Hospital, Chinese Academy of Medical Science and Peking Union Medical College, No. I Shuaifuyuan, Dongcheng District, Beijing, 100730, People's Republic of China

Tel/Fax +86 1069155073

Email t75y@sina.com; xiehezhaoweigang@163. com
Objective: This study aimed to find the association between alanine transaminase-toaspartate aminotransferase ratio (ALT/AST) and the incidence of gestational diabetes mellitus (GDM).

Methods: A total of 1128 pregnant women were included in this prospective, double-center, observational cohort study. ALT, AST and total bilirubin (TBil) were tested during 6-12 weeks of gestation and 75-g oral glucose tolerance test (OGTT) was conducted during 24-28 weeks of gestation to screen GDM. The association between ALT/AST and glucose concentration during OGTT was analyzed by linear regression model. The OR with $95 \% \mathrm{CI}$ for incidence of GDM associated with ALT/AST was estimated by binary logistic regression. The discriminatory values of ALT/AST and triglyceride/high-density lipoprotein cholesterol (TG/HDL-C) for GDM were calculated by the area under the receiver operating characteristic curve (ROC-AUC).

Results: The incidence of GDM was $22.07 \%$ (249/1128). ALT/AST was higher in GDM group than in NGT group $(0.92[0.75,1.18]$ vs $0.80[0.65,1.02], P<0.001)$. ALT/AST had positive correlations with fasting blood glucose, 1-hour and 2-hour blood glucose concentration during OGTT (0.089 [95\% CI: 0.034, 0.163], 0.176 [95\% CI: $0.052,0.104$ ], and 0.115 [95\% CI: 0.199, 0.609], respectively). The OR of ALT/AST for incidence of GDM was 1.603 (95\% CI:1.097, 2.344). The ROC-AUC of ALT/AST and TG/HDL-C reached 0.615 (95\% CI: $0.575,0.655)$ and 0.619 (95\% CI: 0.580, 0.659), respectively.

Conclusion: ALT/AST in early pregnancy was an independent risk factor of GDM. The predictive ability of ALT/AST was similar to TG/HDL-C for GDM.

Keywords: gestational diabetes mellitus, type 2 diabetes mellitus, alanine transaminase, aspartate aminotransferase, triglyceride

\section{Introduction}

Gestational diabetes mellitus (GDM) is defined as a state of impaired glucose tolerance first being discovered during pregnancy. ${ }^{1}$ The prevalence of GDM has increased sharply worldwide and the increase of incidence of GDM in China has been especially alarming in the past few years. ${ }^{2}$ As one of the most common medical complications of pregnancy, GDM increases the risk of pregnancy complications and adverse perinatal outcomes, such as pregnancy-induced hypertension, preeclampsia, abortion, premature delivery, premature ruptures of membranes, large for gestational age infant and so on. It also increases the risk of type 2 diabetes (T2DM) for mother in future and influences the metabolism of offspring 
in the long run, ${ }^{3,4}$ bringing both public health and economic burden. Thus more and more studies have focused on finding risk factors of GDM, which may help predict and prevent GDM.

Non-alcoholic fatty liver disease (NAFLD) is the most common chronic liver disease in the world and more than $25 \%$ of the world's population is currently thought to have NAFLD. ${ }^{5}$ It is considered as a hepatic manifestation of metabolic syndrome or insulin resistance. Substantial evidences have shown that NAFLD is associated with impaired glucose homeostasis and higher risk of GDM. ${ }^{6,7}$ Aspartate aminotransferase-to-alanine transaminase ratio (AST/ALT) was first proposed by Fernando De Ritis in 1957 to evaluate the severity of viral hepatitis, which was also called De Ritis ratio. ${ }^{8}$ Since then ALT/AST or AST/ALT has been reported in lots of studies and has been found to be not only an assessment tool of hepatic diseases, but also a valuable prognostic tool for cardiovascular disease, ${ }^{9}$ arterial stiffness, ${ }^{10}$ cancers, ${ }^{11,12}$ chronic kidney disease ${ }^{13}$ and so on. Recently it has been of great interest as a possible novel marker for metabolic syndrome ${ }^{14,15}$ and insulin resistance. ${ }^{16}$ Zou et al ${ }^{17}$ found ALT/AST was closely related to the risk of new-onset NAFLD. Thus we speculated ALT/AST might be a risk factor of GDM. However, to our best knowledge, no study to date has investigated ALT/AST in early pregnancy among patients with subsequent risk of GDM. Therefore, we examined the association between ALT/AST in early pregnancy and the incidence of GDM in a prospective, double-center, observational cohort study among Chinese pregnant women and evaluated the predictive ability of ALT/AST for GDM. Since triglyceride-to-high-density lipoprotein cholesterol ratio (TG/HDL-C) has been reported as an effective predictor of GDM, the predictive ability of ALT/AST was compared with that of TG/HDL-C. ${ }^{18,19}$

\section{Materials and Methods}

This is a part of an ongoing prospective, double-center, observational cohort study which aimed to find reliable diagnostic markers of GDM in early pregnancy by urinary proteomics. The study started in 2019 in Haidian District Maternal and Child Health Care Hospital and Chaoyang District Maternal and Child Health Care Hospital, Beijing, China. All participants underwent a clinical investigation at the first prenatal visit in early pregnancy (6-12 weeks of gestation). ${ }^{20}$

\section{Study Participants}

Inclusion and exclusion criteria of participants were as follows. Inclusion criteria: (1) gestation age at entry $<12$ weeks;
(2) without impaired glucose tolerance or diabetes mellitus before pregnancy; (3) acceptance of participation in the study and signature of the consent form. Exclusion criteria: (1) gemellary or multiple pregnancy; (2) fasting blood glucose $\geq 6.1 \mathrm{mmol} / \mathrm{L}$ at baseline; (3) any acute or other severe chronic diseases, such as autoimmune disease, severe liver or renal dysfunction, heart disease and so on. On this basis, a total of 1128 pregnant women with clinical and laboratory data at 612 weeks of gestation and 75 -g oral glucose tolerance test (OGTT) at 24-28 weeks of gestation were enrolled in the present study from Aug, 2019 to Nov, 2020.

The ethics committees of all participating centers approved the study protocol. The study was conducted under the guidance of Major New Drugs Innovation and Development Program (clinical trial number is NCT03246295). Written informed consent was obtained from each participant and the study was performed in accordance with the Declaration of Helsinki as revised in 2013.

\section{Measurements}

Participants were measured for body weight, height, systolic blood pressure (SBP) and diastolic blood pressure (DBP) at the first prenatal visit (6-12 weeks of gestation). SBP and DBP were taken by trained nurses with an automatic blood pressure monitor. Body mass index (BMI) was determined by dividing body weight in kilograms by height in meters squared. Medical history, personal history and family history were asked by attending doctors and recorded in electronic medical records (EMRs).

Blood samples were collected at the first prenatal visit and 75-g OGTT was conducted for all participants during 24-28 weeks of gestation. Biochemical parameters presented in the present study, including ALT, AST, total bilirubin (TBil), total cholesterol (TC), TG, HDL-C and low-density lipoprotein cholesterol (LDL-C), were measured by Beckman AU5800 automatic analyzer. Quality controls were performed by Bio-Rad biochemical quality control product.

GDM was diagnosed according to the International Association of the Diabetes and Pregnancy Study Group's criterion in $2010 .{ }^{1}$ For all participants in the present study, all available clinical and laboratory data were recorded and verified by two researchers at the same time.

\section{Statistical Analysis}

Analyses were conducted by the statistical program SPSS (version 24, SPSS, Chicago, IL). Continuous variables were tested for normality of distribution. Variables with 
approximately normal distributions were presented as mean \pm SD and those with skewed distributions were presented as median and interquartile range (25th-75th percentile). Categorical variables were presented as percentages (numbers). A two-sample Student $t$ test or Mann-Whitney test was used for continuous variables and $\chi 2$ test was used for categorical variables. The association between ALT/AST and glucose concentration at different points during OGTT was analyzed by linear regression. Stepwise binary logistic regression was applied to examine the relevance between ALT/AST and GDM. Maternal age, preBMI, FHDM and blood lipids, which were known as risk factors of GDM, were considered as confounding factors in the present study. TC was excluded to avoid the multi-collinearity issue (VIF $>5$ ). The area under the receiver operating characteristic curve (ROCAUC) was used to evaluate the predictive ability of ALT/ AST for GDM. With Bonferroni correction for multiple comparisons of blood glucose at three time points during 75-g OGTT, the prespecified level of statistical significance was set as $P$-values of $<0.017$ (ie, 0.05 divided by 3). Statistical significance was inferred from twosided $P$-values of $<0.05$ for other analyses.

\section{Results}

The incidence of GDM was 22.07\% (249/1128) in this study. Table 1 showed anthropometric and biochemical

Table I Anthropometric and Biochemical Characteristics Between Women with and without GDM

\begin{tabular}{|c|c|c|c|}
\hline Variables & GDM $(n=249)$ & NGT $(n=879)$ & $P$-value \\
\hline Age & $31.43 \pm 3.91$ & $30.11 \pm 3.93$ & $<0.001$ \\
\hline PreBMI $\left(\mathrm{kg} / \mathrm{m}^{2}\right)$ & $22.80(20.40,25.46)$ & $21.45(19.72,23.15)$ & $<0.001$ \\
\hline FHDM & $18.1 \%(45)$ & II.0\%(97) & 0.003 \\
\hline $\mathrm{SBP}(\mathrm{mmH})$ & $114.7 \pm 9.53$ & $112.5 \pm 10.45$ & 0.005 \\
\hline $\mathrm{DBP}(\mathrm{mmHg})$ & $71.2 \pm 8.48$ & $70.52 \pm 9.07$ & 0.295 \\
\hline $\mathrm{ALT}(\mathrm{U} / \mathrm{L})$ & $15.00(\mid 1.00,20.45)$ & $12.60(10.00,17.69)$ & $<0.001$ \\
\hline AST (U/L) & $16.17(14.00,19.00)$ & $16.00(14.00,18.70)$ & 0.444 \\
\hline TBil (umol/L) & $10.40(8.50,13.40)$ & $10.50(8.40,13.00)$ & 0.809 \\
\hline $\mathrm{TC}(\mathrm{mmol} / \mathrm{L})$ & $4.09(3.53,4.70)$ & $3.98(3.49,4.52)$ & 0.074 \\
\hline $\mathrm{TG}(\mathrm{mmol} / \mathrm{L})$ & $0.96(0.73,1.30)$ & $0.85(0.65,1.10)$ & $<0.001$ \\
\hline HDL-C (mmol/L) & $1.43(1.19,1.61)$ & $\mathrm{I} .50(\mathrm{I} .29, \mathrm{I} .72)$ & 0.001 \\
\hline LDL-C (mmol/L) & $2.15(1.77,2.72)$ & $1.99(1.69,2.43)$ & $<0.001$ \\
\hline ALT/AST & $0.92(0.75,1.18)$ & $0.80(0.65,1.02)$ & $<0.001$ \\
\hline TG/HDL-C & $0.69(0.51,0.94)$ & $0.56(0.42,0.77)$ & $<0.001$ \\
\hline
\end{tabular}

Abbreviations: GDM, gestational diabetes mellitus; NGT, normal glucose tolerance; preBMI, pre-pregnancy body mass index; FHDM, family history of diabetes mellitus; SBP, systolic blood pressure; DBP, diastolic blood pressure; ALT, alanine transaminase; AST, aspartate aminotransferase; TBil, total bilirubin; TC, total cholesterol; TG, triglyceride; HDL-C, high-density lipoprotein cholesterol; LDL-C, lowdensity lipoprotein cholesterol. characteristics in women with GDM and normal glucose tolerance (NGT). Maternal age, pre-pregnancy body mass index (preBMI), percentage of family history of diabetes mellitus (FHDM), SBP, ALT, TG and LDL-C in GDM group were significantly higher than those in NGT group $(P<0.05)$. ALT/AST and TG/HDL-C were also significantly higher in GDM group $(0.92[0.75,1.180]$ vs 0.80 [0.65, 1.02], $P<0.001 ; 0.69$ [0.51, 0.94] vs 0.56 [0.42, $0.77], P<0.001$, respectively).

Linear regression analysis was performed to estimate the relationship between ALT/AST and glucose concentration at three time points of OGTT (Table 2). There were positive correlations between ALT/AST and fasting blood glucose (FBG), 1-hour blood glucose (1-h BG) as well as 2-h BG $(P<0.001)$. And after adjustment for confounding factors, including age, preBMI, FHDM and blood lipids, the positive correlations were still statistically significant $(P<0.017)$.

Table 3 presented the crude and multivariable-adjusted OR of GDM determined by ALT/AST. There was higher risk for the incidence of GDM with increasing ALT/AST (OR [95\% CI] 2.143 [1.500, 3.061]). The OR for the incidence of GDM was (OR [95\% CI] 1.623 [1.116,

Table 2 Association of ALT/AST and Glucose Concentration at Three Time Points During OGTT at 24-28 Weeks' Gestation

\begin{tabular}{|l|c|c|c|c|}
\hline \multirow{2}{*}{$\begin{array}{l}\text { OGTT } \\
\text { (mmol/L) }\end{array}$} & \multicolumn{2}{|c|}{ Unadjusted } & \multicolumn{2}{c|}{ Fully Adjusted $^{\#}$} \\
\cline { 2 - 5 } & $\beta(95 \% \mathbf{C l})$ & $P$-value & $\beta(95 \% \mathbf{C l})$ & P-value \\
\hline FBG & $0.120(0.069,0.198)$ & $<0.00 I^{*}$ & $0.089(0.034,0.163)$ & $0.003^{*}$ \\
I-h BG & $0.134(0.346,0.894)$ & $<0.00 I^{*}$ & $0.176(0.052,0.104)$ & $0.002^{*}$ \\
2-h BG & $0.151(0.327,0.738)$ & $<0.00 I^{*}$ & $0.115(0.199,0.609)$ & $<0.00 I^{*}$ \\
\hline
\end{tabular}

Notes: *A priori statistical significance is $P<0.017$. ${ }^{*}$ Adjusted for age, prepregnancy body mass index, family history of diabetes mellitus, triglyceride, highdensity lipoprotein cholesterol and low-density lipoprotein cholesterol. Abbreviations: GDM, gestational diabetes mellitus; ALT, alanine transaminase; AST, aspartate aminotransferase; OGTT, oral glucose tolerance test; FBG, fasting blood glucose.

Table 3 Odds Ratios for GDM in Relation to ALT/AST

\begin{tabular}{|c|c|c|c|c|}
\hline & $\boldsymbol{\beta}$ & OR & $95 \% \mathbf{C I}$ & $\boldsymbol{P}$-value \\
\hline Model I & 0.762 & 2.143 & $1.500,3.06 \mathrm{I}$ & $<0.00 \mathrm{I}^{*}$ \\
Model 2 & 0.484 & 1.623 & $1.116,2.360$ & $0.01 \mathrm{I}^{*}$ \\
Model 3 & 0.472 & 1.603 & $1.097,2.344$ & $0.015^{*}$ \\
\hline
\end{tabular}

Notes: $* p<0.05$ represents statistical significance. Model I: ALT/AST. Model 2: Model I + age, pre-pregnancy body mass index, family history of diabetes mellitus. Model 3: Model 2 + triglyceride, high-density lipoprotein cholesterol, low-density lipoprotein cholesterol.

Abbreviations: GDM, gestational diabetes mellitus; ALT, alanine transaminase; AST, aspartate aminotransferase. 
2.360]) after adjustment for age, preBMI and FHDM. After further adjustment for blood lipids in the second model, the association was still significant (OR [95\% CI] $1.603[1.097,2.344])$.

ROC curves were used to evaluate the predictive ability of ALT/AST and TG/HDL-C for GDM (Figure 1). The cut-off point of ALT/AST for predicting GDM in early pregnancy was 0.825 (sensitivity $67.1 \%$ and specificity $54.2 \%$; ROC-AUC $0.615, \quad 95 \%$ CI $0.575-0.655$, $P<0.001$ ). Whereas the cut-off point of TG/HDL-C was 0.676 (sensitivity $51.4 \%$ and specificity $68.3 \%$; ROCAUC $0.619,95 \%$ CI $0.580-0.659, P<0.001)$. Z test performed by MedCalc showed that the predictive ability of ALT/AST for GDM was similar to TG/HDL-C (z statistic $0.144, P=0.89$ ). The ROC-AUC of ALT was the lowest among them (AUC 0.597, 95\% CI 0.577-0.637, $P<0.001)$.

\section{Discussion}

In this study, we investigated the association between the ALT/AST in early pregnancy and the incidence of GDM, and we also reported a positive relationship between ALT/ AST and glucose concentration at three time points during OGTT. The present analyses implied ALT/AST was an independent risk factor of GDM and it appeared to have a similar predictive ability for GDM compared with TG/ HDL-C in this cohort study. These results were still statistically significant after adjustment for potential

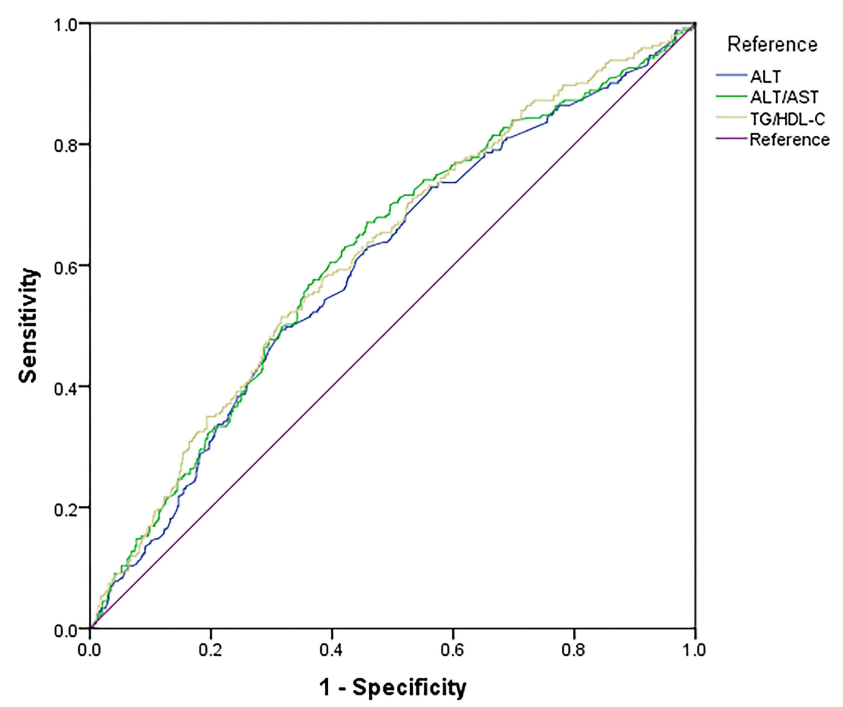

Figure I Comparison of ALT/AST, TG/HDL-C and ALT as risk makers of GDM. Abbreviations: GDM, gestational diabetes mellitus; ALT, alanine transaminase; AST, aspartate aminotransferase; TG, triglyceride; HDL-C, high-density lipoprotein cholesterol. confounding factors. To the best of our knowledge, this study is the first to report the relationship between ALT/ AST and GDM.

The liver minimizes the fluctuation of postprandial blood glucose by taking up and storing glucose and 60 $65 \%$ oral glucose load is disposed of by liver thus any impairment of liver may affect postprandial glycemia. ${ }^{21}$ Previous studies examined association between ALT/AST and glucose concentration during 75-g OGTT and found the significant change was only noted in 2-h BG between subjects with normal and abnormal ALT/AST. ${ }^{22}$ In this study, although ALT and AST were both in normal range, the analysis showed there were positive correlations between ALT/AST and glucose concentration at different time points during OGTT. After adjustment for key confounding factors, there were still significant correlations.

Previous studies reported the close relationship between T2DM and several liver enzymes, including gamma glutamyl transferase (GGT), ALT and AST. ${ }^{23}$ But the association between different liver enzymes and GDM were still controversial. Most previous studies reported that higher GGT and ALT were risk factors for GDM and that AST may not be related to GDM. A recent metaanalysis implied only GGT presented significant positive association with GDM. ${ }^{24}$ As a hepatic marker first reported to be an assessment tool for viral hepatic diseases, ALT/AST has been found to be associated with metabolic diseases recently. Pinnaduwage et $\mathrm{al}^{25}$ evaluated the impact of changes in different single liver enzyme as well as ALT/AST on changes in insulin sensitivity, $\beta$-cell function and glycemia in 336 women with varying degrees of previous gestational glucose metabolic status at 1 and 3 years postpartum and found ALT/AST rather than single liver enzyme could track changes in insulin sensitivity and $\beta$-cell function. Compared with the single variable, ALT/ AST may amplify a signal of GDM risk that is not yet detectable with individual liver enzyme alone. However, previous studies did not examine the relationship between ALT/AST and GDM during pregnancy. In the present study, although there was no significant difference of AST concentration between two groups, the results showed ALT/AST could predict GDM more accurately than using ALT alone, implying AST maybe also have slight association with GDM.

The mechanism of the relationship between ALT/AST and GDM was unclear but there are several possible speculations. The liver plays an important role in controlling glycogenesis, glycogenolysis, glycolysis and 
gluconeogenesis, which are key pathways of maintaining glucose homeostasis. ${ }^{26}$ Any impairment of liver may influence this process while ALT and AST both have strong positive correlation with liver fat accumulation. ${ }^{27,28}$ As liver fat is a key feature of insulin resistance and NAFLD, higher ALT/AST may imply relatively worse metabolic status. The positive correlation of liver enzymes and insulin resistance has been reported ${ }^{29}$ and previous studies suggested ALT/AST was a good predictor of insulin resistance in Asian populations. ${ }^{16,30}$ NAFLD has mutual influence with $\mathrm{GDM}^{7}$ and a study among nonobese Chinese population without NAFLD at baseline reported the increase in the ALT/AST was associated with the risk of new-onset NAFLD. ${ }^{17}$ Bettini et $\mathrm{al}^{31}$ found ALT/AST decreased in patients with obesity after laparoscopic sleeve gastrectomy and was correlated with BMI and insulin reduction. Since insulin resistance, NAFLD and BMI are all closely related to the development of GDM, it is reasonable that ALT/AST presented close association with GDM and can be an independent risk factor of GDM. This result also evidenced the relationship between NAFLD and GDM from another perspective by a simple index.

TG/HDL-C has been reported as a good predictor for GDM in some studies. ${ }^{18,19}$ It is acknowledged TG is closely associated with diet in the short term so a single test during first trimester with great variability may not be stable enough. Besides, liver function is a routine examination whereas lipids are not tested in prenatal examination in some regions in China. In this study, we found the predictive ability of ALT/AST for GDM was similar to that of TG/HDL-C. Therefore, as an independent risk factor of GDM, ALT/AST was a more stable and easily available index than TG/HDL-C and is worth investigating in further studies. However, we also noticed that the predictive ability of TG/HDL-C and ALT/AST were both pretty limited. Although the predictive ability of ALT/ AST for GDM has not been reported and was unclear, the performance of TG/HDL-C was not consistent with previous studies. There are several potential explanations. First, there might be publication bias because usually only positive results with good predictive ability of TG/HDL-C for GDM were reported. Second, pregnant women enrolled in the study may get more attention from doctors, which may lower the predictive ability of some parameters in early gestation. Moreover, the complicated mechanisms of the development of GDM make it difficult to predict such development accurately with a single index; however, more investigation on different risk factors of GDM is beneficial to further understanding of GDM and can contribute to build better prediction models in future.

There were some other limitations in this study. First, some potential confounding factors such as information on education, occupation, income and eating habits were not collected. Second, although women with severe hepatic diseases were not enrolled in this study, the ALT/AST might have been biased by the presence of an undetected liver disease. Notwithstanding these limitations, the current analyses were adjusted for major risk factors of GDM, including age, preBMI, FHDM and blood lipids, to decrease bias. These factors are acknowledged to have significant effects on GDM and significant differences between women with and without GDM also existed in this study. As an independent risk factor of GDM, ALT/ AST is a simple, economical and easily available index. It could be a potentially valuable component of a prediction model of GDM and deserves to be further investigated. Expanding the sample size in different races and extending the follow-up duration will help further clarify the relationship between ALT/AST and incidence of GDM as well as risk of T2DM in future among women with GDM history.

\section{Conclusion}

In summary, higher ALT/AST in early pregnancy, even within the normal range, was an independent risk factor of GDM. ALT/AST presented similar predictive ability to TG/HDL-C for GDM in this cohort study.

\section{Data Sharing Statement}

The datasets used and/or analyzed during the current study are available from the corresponding author on reasonable request.

\section{Ethics Approval}

The ethics committees of all participating centers approved the study protocol (Peking Union Medical College Hospital [NO. JS-2574], Institute of Beijing Medical Science Chinese Academy of Medical Sciences [NO. 047-2019], Haidian District Maternal and Child Health Care Hospital [NO. 2019-17] and Beijing Chaoyang District Maternal and Child Health Care Hospital licensed by National Center for Women and Children's Health [NO. FY2019-01]). The study was performed in accordance with the Declaration of Helsinki as revised in 2013. 


\section{Consent}

All participants had given written consent to the inclusion of material pertaining to themselves and acknowledged they had been fully anonymized so cannot be identified via the paper.

\section{Acknowledgments}

The authors thank all the participants in this study.

\section{Funding}

This study was supported by "13th Five-Year" National Science and Technology Major Project for New Drugs under Grant No. 2019ZX09734001 (to Weigang Zhao).

\section{Disclosure}

All authors declare that there is no conflict of interest regarding the publication of this paper.

\section{References}

1. Metzger BE, Gabbe SG, Persson B, et al. International association of diabetes and pregnancy study groups recommendations on the diagnosis and classification of hyperglycemia in pregnancy. Diabetes Care. 2010;33(3):676-682. doi:10.2337/dc09-1848

2. Gao C, Sun X, Lu L, Liu F, Yuan J. Prevalence of gestational diabetes mellitus in mainland China: a systematic review and meta-analysis. J Diabetes Investig. 2019;10(1):154-162. doi:10.1111/jdi.12854

3. Billionnet C, Mitanchez D, Weill A, et al. Gestational diabetes and adverse perinatal outcomes from 716,152 births in France in 2012. Diabetologia. 2017;60(4):636-644. doi:10.1007/s00125-017-4206-6

4. Damm P, Houshmand-Oeregaard A, Kelstrup L, Lauenborg J, Mathiesen ER, Clausen TD. Gestational diabetes mellitus and long-term consequences for mother and offspring: a view from Denmark. Diabetologia. 2016;59(7):1396-1399. doi:10.1007/s00125-016-3985-5

5. Younossi Z, Tacke F, Arrese M, et al. Global perspectives on nonalcoholic fatty liver disease and nonalcoholic steatohepatitis. Hepatology. 2019;69(6):2672-2682. doi:10.1002/hep.30251

6. Lee SM, Kwak SH, Koo JN, et al. Non-alcoholic fatty liver disease in the first trimester and subsequent development of gestational diabetes mellitus. Diabetologia. 2019;62(2):238-248. doi:10.1007/s00125-0184779-8

7. De Souza LR, Berger H, Retnakaran R, et al. Non-alcoholic fatty liver disease in early pregnancy predicts dysglycemia in mid-pregnancy: prospective Study. Am J Gastroenterol. 2016;111 (5):665-670. doi:10.1038/ajg.2016.43

8. De Ritis F, Coltorti M, Giusti G. An enzymic test for the diagnosis of viral hepatitis: the transaminase serum activities. 1957. Clin Chim Acta. 2006;369(2):148-152. doi:10.1016/j.cca.2006.05.001

9. Weng SF, Kai J, Guha IN, Qureshi N. The value of aspartate aminotransferase and alanine aminotransferase in cardiovascular disease risk assessment. Open Heart. 2015;2(1):e000272. doi:10.1136/ openhrt-2015-000272

10. Liu Y, Zhao P, Cheng M, et al. AST to ALT ratio and arterial stiffness in non-fatty liver Japanese population: a secondary analysis based on a cross-sectional study. Lipids Health Dis. 2018;17(1):275. doi:10.1186/s12944-018-0920-4

11. Quhal F, Abufaraj M, Janisch F, et al. The significance of De Ritis ratio in patients with radiation-recurrent prostate cancer undergoing salvage radical prostatectomy. Arab J Urol. 2020;18(4):213-218. doi:10.1080/2090598x.2020.1771947
12. Wagner-Johnston ND, Sharman J, Furman RR, et al. Idelalisib immune-related toxicity is associated with improved treatment response. Leuk Lymphoma. 2021:1-6. doi:10.1080/10428194.20 21.1948038

13. Ochiai H, Shirasawa T, Yoshimoto T, et al. Elevated alanine aminotransferase and low aspartate aminotransferase/alanine aminotransferase ratio are associated with chronic kidney disease among middle-aged women: a cross-sectional study. BMC Nephrol. 2020;21(1):471. doi:10.1186/s12882-020-02144-6

14. Yadav D, Choi E, Ahn SV, et al. Incremental predictive value of serum AST-to-ALT ratio for incident metabolic syndrome: the ARIRANG Study. PLoS One. 2016;11(8):e0161304. doi:10.1371/ journal.pone.0161304

15. Warnakulasuriya LS, Samaranayake DL, Adikaram AVN, et al. Metabolic abnormalities in a cohort of overweight and obese children in an urban setting of Sri Lanka. Int $J$ Endocrinol. 2021; 2021:9936889. doi:10.1155/2021/9936889

16. Kawamoto R, Kohara K, Kusunoki T, Tabara Y, Abe M, Miki T. Alanine aminotransferase/aspartate aminotransferase ratio is the best surrogate marker for insulin resistance in non-obese Japanese adults. Cardiovasc Diabetol. 2012;11:117. doi:10.1186/1475-2840-11-117

17. Zou Y, Zhong L, Hu C, Sheng G. Association between the alanine aminotransferase/aspartate aminotransferase ratio and new-onset non-alcoholic fatty liver disease in a nonobese Chinese population: a population-based longitudinal study. Lipids Health Dis. 2020;19 (1):245. doi:10.1186/s12944-020-01419-z

18. Liu PJ, Liu Y, Ma L, et al. The predictive ability of two triglyceride-associated indices for gestational diabetes mellitus and large for gestational age infant among Chinese pregnancies: a Preliminary Cohort Study. Diabetes Metab Syndr Obes. 2020;13:2025-2035. doi:10.2147/dmso.S251846

19. Wang D, Xu S, Chen H, Zhong L, Wang Z. The associations between triglyceride to high-density lipoprotein cholesterol ratios and the risks of gestational diabetes mellitus and large-for-gestational-age infant. Clin Endocrinol (Oxf). 2015;83(4):490-497. doi:10.1111/cen.12742

20. Song S, Zhang Y, Qiao X, et al. HOMA-IR as a risk factor of gestational diabetes mellitus and a novel simple surrogate index in early pregnancy. Int J Gynaecol Obstet. 2021. doi:10.1002/ijgo.13905

21. Moore MC, Coate KC, Winnick JJ, An Z, Cherrington AD. Regulation of hepatic glucose uptake and storage in vivo. Adv Nutr. 2012;3(3):286-294. doi:10.3945/an.112.002089

22. Su CC, Wang K, Hsia TL, Chen CS, Tung TH. Association of nonalcoholic fatty liver disease with abnormal aminotransferase and postprandial hyperglycemia. J Clin Gastroenterol. 2006;40(6):5 51-554. doi:10.1097/00004836-200607000-00015

23. Zhang J, Cheng N, Ma Y, et al. Liver enzymes, fatty liver and type 2 diabetes mellitus in a jinchang cohort: a prospective study in adults. Can J Diabetes. 2018;42(6):652-658. doi:10.1016/j.jcjd.2018.02. 002

24. Zhao W, Zhang L, Zhang G, et al. The association of plasma levels of liver enzymes and risk of gestational diabetes mellitus: a systematic review and dose-response meta-analysis of observational studies. Acta Diabetol. 2020;57(6):635-644. doi:10.1007/s00592-019-014 58-8

25. Pinnaduwage L, Ye C, Hanley AJ, et al. Changes over time in hepatic markers predict changes in insulin sensitivity, $\beta$-cell function, and glycemia. J Clin Endocrinol Metab. 2018;103(7):2651-2659. doi:10. 1210/jc.2018-00306

26. Han HS, Kang G, Kim JS, Choi BH, Koo SH. Regulation of glucose metabolism from a liver-centric perspective. Exp Mol Med. 2016;48 (3):e218. doi:10.1038/emm.2015.122

27. Pirimoğlu B, Sade R, Polat G, Işlek A, Kantarcı M. Analysis of correlation between liver fat fraction and AST and ALT levels in overweight and obese children by using new magnetic resonance imaging technique. Turk $J$ Gastroenterol. 2020;31(2):156-162. doi:10.5152/tjg.2020.18594 
28. Netaji A, Jain V, Gupta AK, Kumar U, Jana M. Utility of MR proton density fat fraction and its correlation with ultrasonography and biochemical markers in nonalcoholic fatty liver disease in overweight adolescents. J Pediatr Endocrinol Metab. 2020;33(4):473-479. doi:10.1515/jpem-2019-0463

29. Uetani E, Tabara Y, Igase M, et al. Liver enzyme and adipocytokine profiles are synergistically associated with insulin resistance: the J-SHIPP study. J Atheroscler Thromb. 2012;19(6):577-584. doi:10. 5551/jat. 11924
30. Zhao L, Cheng J, Chen Y, et al. Serum alanine aminotransferase/ aspartate aminotransferase ratio is one of the best markers of insulin resistance in the Chinese population. Nutr Metab. 2017;14:64. doi:10.1186/s12986-017-0219-x

31. Bettini S, Bordigato E, Milan G, et al. SCCA-IgM as a potential biomarker of non-alcoholic fatty liver disease in patients with obesity, prediabetes and diabetes undergoing sleeve gastrectomy. Obes Facts. 2019;12(3):291-306. doi:10.1159/000499717

\section{Publish your work in this journal}

The International Journal of General Medicine is an international, peer-reviewed open-access journal that focuses on general and internal medicine, pathogenesis, epidemiology, diagnosis, monitoring and treatment protocols. The journal is characterized by the rapid reporting of reviews, original research and clinical studies across all disease areas. The manuscript management system is completely online and includes a very quick and fair peer-review system, which is all easy to use. Visit http://www.dovepress.com/ testimonials.php to read real quotes from published authors.

Submit your manuscript here: https://www.dovepress.com/international-journal-of-general-medicine-journal 